\title{
On the arithmetic of certain modular curves
}

\author{
by \\ Daeyeol Jeon (Kongju) and Chang Heon Kim (Seoul)
}

0. Introduction. Let $N$ be a positive integer and $\Delta$ a subgroup of $(\mathbb{Z} / N \mathbb{Z})^{*}$ which contains \pm 1 . Let $X_{\Delta}(N)$ be the modular curve defined over $\mathbb{Q}$ associated to the congruence subgroup

$$
\Gamma_{\Delta}(N):=\left\{\left(\begin{array}{ll}
a & b \\
c & d
\end{array}\right) \in \mathrm{SL}_{2}(\mathbb{Z})|a \bmod N \in \Delta, N| c\right\} .
$$

Then all the intermediate modular curves between $X_{1}(N)$ and $X_{0}(N)$ are of the form $X_{\Delta}(N)$. Denote the genus of $X_{\Delta}(N)$ by $g_{\Delta}(N)$. In this paper we study the arithmetic of the curves $X_{\Delta}(N)$.

In Section 1 we prove a genus formula for the curves $X_{\Delta}(N)$ which was referred to in the authors' previous works [J-K1, J-K2, J-K-S] without proof.

A smooth projective curve $X$ defined over an algebraically closed field $k$ is called $d$-gonal if it admits a map $\phi: X \rightarrow \mathbb{P}^{1}$ over $k$ of degree $d$. For $d=3$ we say that the curve is trigonal. Also, the smallest possible $d$ is called the gonality of the curve and is denoted by $\operatorname{Gon}(X)$.

Hasegawa and Shimura [H-S1] proved that $X_{0}(N)$ is trigonal if and only if it is of genus $g \leq 2$ or is not hyperelliptic of genus $g=3,4$. In fact the "if" part is well-known. The modular curves $X_{0}(N)$ carry the action of the Atkin-Lehner involutions $W_{d}$ for any $d \| N$, i.e., for any positive integer $d$ dividing $N$ with $(d, N / d)=1$. Let $X_{0}^{+d}(N)$ and $X_{0}^{*}(N)$ be the quotients of $X_{0}(N)$ by $W_{d}$ and by the $W_{d}$ 's for all $d \| N$ respectively. In [H-S2, H-S3], Hasegawa and Shimura also determined the trigonal modular curves $X_{0}^{+d}(N)$ and $X_{0}^{*}(N)$, and found that there exist non-trivial trigonal modular curves, i.e., those of genus $g \geq 5$.

The authors and Schweizer [J-K-S] showed that there exist no non-trivial trigonal modular curves $X_{1}(N)$, which plays a central role in determining the torsion structures of elliptic curves defined over cubic number fields; such structures occur infinitely often.

2000 Mathematics Subject Classification: 11G18, 11G30.

Key words and phrases: genus, hyperelliptic, trigonal, modular curve. 
In Section 3 we determine all the intermediate modular curves between $X_{1}(N)$ and $X_{0}(N)$ which are trigonal, and conclude that there exist no non-trivial trigonal curves. For this purpose, it is necessary to determine all the hyperelliptic intermediate modular curves, which was done by Ishii and Momose $[\mathrm{I}-\mathrm{M}]$. In fact, they claimed that there existed no such modular curves. But we find that there is a unique hyperelliptic intermediate modular curve, namely $X_{\Delta_{1}}(21)$ (see Theorem 2.3). As Enrique González-Jiménez pointed out, the "lost" curve $X_{\Delta_{1}}(21)$ is a new hyperelliptic curve in the sense of [B-G-G-P] and it is the curve labeled $C_{21 A_{\{0,2\}}}^{A}$ with equation $y^{2}=$ $\left(x^{2}-x+1\right)\left(x^{6}+x^{5}-6 x^{4}-3 x^{3}+14 x^{2}-7 x+1\right)$.

1. A genus formula. Let $\Gamma(1)=\mathrm{SL}_{2}(\mathbb{Z})$ be the full modular group. For any integer $N \geq 1$, we have the subgroups $\Gamma_{1}(N)$ and $\Gamma_{0}(N)$ of $\Gamma(1)$ consisting of the matrices $\left(\begin{array}{ll}a & b \\ c & d\end{array}\right)$ congruent modulo $N$ to $\left(\begin{array}{ll}1 & * \\ 0 & 1\end{array}\right)$ and $\left(\begin{array}{l}* * \\ 0\end{array}\right)$ respectively. We let $X_{1}(N)$ and $X_{0}(N)$ be the modular curves defined over $\mathbb{Q}$ associated to $\Gamma_{1}(N)$ and $\Gamma_{0}(N)$ respectively. The $X$ 's are compact Riemann surfaces. Let $g_{0}(N)$ denote the genus of $X_{0}(N)$. For any congruence subgroup $\Gamma \subset \Gamma(1)$, we shall denote by $\bar{\Gamma}$ the image of $\Gamma$ under the natural map $\Gamma(1) \rightarrow \bar{\Gamma}(1):=\Gamma(1) /\{ \pm 1\}$.

For $d \mid N$, let $\pi_{d}:(\mathbb{Z} / N \mathbb{Z})^{*} \rightarrow(\mathbb{Z} /\{d, N / d\} \mathbb{Z})^{*}$ be the natural projection, where $\{d, N / d\}$ is the least common multiple of $d$ and $N / d$. Then we have the following genus formula:

THEOREM 1.1. The genus of the modular curve $X_{\Delta}(N)$ is given by

$$
g_{\Delta}(N)=1+\frac{\mu}{12}-\frac{\nu_{2}}{4}-\frac{\nu_{3}}{3}-\frac{\nu_{\infty}}{2}
$$

where

$$
\begin{aligned}
\mu & =N \prod_{\substack{p \mid N \\
\text { prime }}}\left(1+\frac{1}{p}\right) \frac{\varphi(N)}{|\Delta|}, \\
\nu_{2} & =\left|\left\{b \bmod N \in \Delta \mid b^{2}+1 \equiv 0 \bmod N\right\}\right| \frac{\varphi(N)}{|\Delta|}, \\
\nu_{3} & =\left|\left\{b \bmod N \in \Delta \mid b^{2}-b+1 \equiv 0 \bmod N\right\}\right| \frac{\varphi(N)}{|\Delta|}, \\
\nu_{\infty} & =\sum_{\substack{d \mid N \\
d>0}} \frac{\varphi(d) \varphi(N / d)}{\left|\pi_{d}(\Delta)\right|} .
\end{aligned}
$$

Proof. We follow the notations of [O1]. One has to check that the index of $\bar{\Gamma}_{\Delta}(N)$ in $\bar{\Gamma}(1)$ is $\mu$, that the number of elliptic fixed points of order 2 (resp. 3) is $\nu_{2}$ (resp. $\nu_{3}$ ), and that the number of cusps is $\nu_{\infty}$. It is easy to 
show that

$$
\mu=\left[\bar{\Gamma}(1): \bar{\Gamma}_{\Delta}(N)\right]=\left[\bar{\Gamma}(1): \bar{\Gamma}_{0}(N)\right]\left[\bar{\Gamma}_{0}(N): \bar{\Gamma}_{\Delta}(N)\right]=N \prod_{p \mid N}\left(1+\frac{1}{p}\right) \frac{\varphi(N)}{|\Delta|} .
$$

Put $L_{0}=\left(\begin{array}{cc}N & 0 \\ 0 & 1\end{array}\right)$. Then the double coset $\Gamma(1) L_{0} \Gamma(1)$ has the right coset decomposition as follows:

$$
\Gamma(1) L_{0} \Gamma(1)=\bigcup \Gamma(1) L
$$

where $L=\left(\begin{array}{ll}a & b \\ 0 & d\end{array}\right)$ with $a>0, a d=N, b$ taken modulo $d$ and $(a, b, d)=1$.

Now we compute $\nu_{2}$ and $\nu_{3}$. Let $A$ be an elliptic element in $\Gamma(1)$ and $P$ the fixed point of $A$ in the complex upper half-plane. Then $P=M P_{0}$ for some $M \in \Gamma(1)$ where $P_{0}=i$ or $e^{2 \pi i / 3}$. Write $L_{0} M=B L$ for some $B \in \Gamma(1)$ and $L=\left(\begin{array}{ll}a & b \\ 0 & d\end{array}\right)$ with $a>0, a d=N$ and $(a, b, d)=1$. Now if $P_{0}=i$, then

$$
\begin{aligned}
A= & M\left(\begin{array}{cc}
0 & -1 \\
1 & 0
\end{array}\right) M^{-1} \in \Gamma_{0}(N)=\Gamma(1) \cap L_{0}^{-1} \Gamma(1) L_{0} \\
& \Leftrightarrow L\left(\begin{array}{cc}
0 & -1 \\
1 & 0
\end{array}\right) L^{-1} \in \Gamma(1) \\
& \Leftrightarrow\left(\begin{array}{ll}
a & b \\
0 & d
\end{array}\right)\left(\begin{array}{cc}
0 & -1 \\
1 & 0
\end{array}\right)\left(\begin{array}{ll}
a & b \\
0 & d
\end{array}\right)^{-1}=\left(\begin{array}{cc}
b / a & -\left(a^{2}+b^{2}\right) / N \\
d / a & b / a
\end{array}\right) \in \Gamma(1) \\
& \Leftrightarrow a=1, d=N \text { and } b^{2}+1 \equiv 0 \bmod N .
\end{aligned}
$$

Similarly if $P_{0}=e^{2 \pi i / 3}$, then

$$
\begin{aligned}
A \in \Gamma_{0}(N) & \Leftrightarrow\left(\begin{array}{ll}
a & b \\
0 & d
\end{array}\right)\left(\begin{array}{cc}
0 & -1 \\
1 & 1
\end{array}\right)\left(\begin{array}{ll}
a & b \\
0 & d
\end{array}\right)^{-1}=\left(\begin{array}{cc}
b / a & -\left(a^{2}-a b+b^{2}\right) / N \\
d / a & (a-b) / a
\end{array}\right) \in \Gamma(1) \\
& \Leftrightarrow a=1, d=N \text { and } b^{2}-b+1 \equiv 0 \bmod N .
\end{aligned}
$$

Write $M=\left(\begin{array}{cc}x & y \\ z & w\end{array}\right)$ and $B=\left(\begin{array}{cc}x^{\prime} & y^{\prime} \\ z^{\prime} & w^{\prime}\end{array}\right)$. From $L_{0} M=B L$ it follows that

$$
\left(\begin{array}{cc}
N x & N y \\
z & w
\end{array}\right)=\left(\begin{array}{ll}
x^{\prime} & b x^{\prime}+N y^{\prime} \\
z^{\prime} & b z^{\prime}+N w^{\prime}
\end{array}\right)
$$

Note that $M\left(\begin{array}{cc}0 & -1 \\ 1 & 0\end{array}\right) M^{-1}=\left(\begin{array}{cc}y w+x z & * \\ * & *\end{array}\right)$ and $M\left(\begin{array}{cc}0 & -1 \\ 1 & 1\end{array}\right) M^{-1}=\left(\begin{array}{c}y w+x z-y z * \\ *\end{array} *\right.$. Then for the elliptic element $A$ of order 2 (resp. 3) to lie in $\Gamma_{\Delta}(N)$, we need $y w+x z \bmod N \in \Delta(\operatorname{resp} . y w+x z-y z \bmod N \in \Delta)$ together with the condition $b^{2}+1 \equiv 0 \bmod N\left(\right.$ resp. $\left.b^{2}-b+1 \equiv 0 \bmod N\right)$. From (1) it is easy to see that $y w+x z \equiv-b \bmod N$ and $y w+x z-y z \equiv-b+1 \bmod N$. Thus if $A$ is an elliptic element of order 2 (resp. 3) in $\bar{\Gamma}_{\Delta}$, then it determines an ele$\operatorname{ment} b \bmod N \in \Delta$ satisfying $b^{2}+1 \equiv 0 \bmod N\left(\operatorname{resp} . b^{2}-b+1 \equiv 0 \bmod N\right)$. 
Conversely, we can form an elliptic element of order 2 (resp. 3) from a solution in $\Delta$ of the congruence equation $x^{2}+1 \equiv 0 \bmod N$ (resp. $x^{2}-x+1$ $\equiv 0 \bmod N)$. We note that different solutions give $\Gamma_{0}(N)$-inequivalent elliptic points of order 2 (resp. 3).

Now we consider the Galois covering $p_{2}: X_{\Delta}(N) \rightarrow X_{0}(N)$. If $A$ is an elliptic element of order 2 in $\bar{\Gamma}_{\Delta}$ and $A P=P$, then each point in the inverse image of $\Gamma_{0}(N) P$ is again an elliptic point of order 2 and has ramification index 1 . Thus the number of elements in $p_{2}^{-1}\left(\Gamma_{0}(N) P\right)$ would become the degree of $p_{2}$, and hence we have the following:

$$
\begin{aligned}
\nu_{2} & =\left|\left\{b \bmod N \in \Delta \mid b^{2}+1 \equiv 0 \bmod N\right\}\right| \cdot \text { degree of } p_{2} \\
& =\left|\left\{b \bmod N \in \Delta \mid b^{2}+1 \equiv 0 \bmod N\right\}\right| \frac{\varphi(N)}{|\Delta|} .
\end{aligned}
$$

Similarly, $\nu_{3}=\left|\left\{b \bmod N \in \Delta \mid b^{2}-b+1 \equiv 0 \bmod N\right\}\right| \varphi(N) /|\Delta|$.

Finally, we compute $\nu_{\infty}$. We follow the notations of [O2]. Let $p_{1}: X_{1}(N)$ $\rightarrow X_{\Delta}(N)$ and $p_{2}: X_{\Delta}(N) \rightarrow X_{0}(N)$ be the Galois coverings and $p=p_{2} \circ p_{1}$. Denote by $s=\left(\begin{array}{l}x \\ y\end{array}\right)$ a cusp in $X_{1}(N)$. Then

$$
e_{p}(s)=e_{p_{1}}(s) e_{p_{2}}\left(p_{1} s\right) \quad \text { and } \quad e_{p}(s)=(N / d, d) \quad \text { with } d=(y, N)
$$

where $e$ 's denote ramification indices ([O2, Proposition 2]). Now we claim that

$$
e_{p_{1}}(s)=|\Delta| /\left|\pi_{d}(\Delta)\right| .
$$

Note that the group $G=\Gamma_{\Delta}(N) / \pm \Gamma_{1}(N)$ is isomorphic to $\Delta /\{ \pm 1\}$. Each element $\left(\begin{array}{ll}a & b \\ c & d\end{array}\right) \in \Gamma_{\Delta}(N)$ acts on $\left(\begin{array}{l}x \\ y\end{array}\right)$ as $\left(\begin{array}{c}a x \\ a^{-1} y\end{array}\right)$. Then $\left(\begin{array}{l}x \\ y\end{array}\right)$ and $\left(\begin{array}{c}a x \\ a^{-1} y\end{array}\right)$ represent the same cusp on $X_{1}(N)$ if and only if $a x \equiv \pm x \bmod d$ and $a y \equiv$ $\pm y \bmod N$, i.e., $a \equiv \pm 1 \bmod d$ and $\bmod N / d$.

Recall that $\{\cdot, \cdot\}$ denotes least common multiple. Let $H=\{a \bmod N \in$ $\Delta /\{ \pm 1\} \mid a \equiv 1 \bmod \{d, N / d\}\}$. Since $H$ is the kernel of the natural map $\Delta /\{ \pm 1\} \rightarrow(\mathbb{Z} /\{d, N / d\})^{*} /\{ \pm 1\}$, the cardinality of $H$ is equal to $|\Delta| /\left|\pi_{d}(\Delta)\right|$. We can view $H$ as a subgroup of $G$. Then $G / H$ has the same cardinality as the set of orbits Gs. Since the elements of $G s$ are the cusps in $X_{1}(N)$ lying over the cusp $p_{1}(s)$ in $X_{\Delta}(N)$, the ramification index of $s$ in $X_{1}(N)$ is equal to the cardinality of $H$. By the claim we come up with

$$
\begin{aligned}
\nu_{\infty} & =\sum_{\substack{d \mid N \\
d>0}} \frac{\operatorname{deg} p_{2}}{e_{p_{2}}} \varphi((d, N / d)) \quad \text { since } p_{2} \text { is a Galois covering } \\
& =\sum_{\substack{d \mid N \\
d>0}} \frac{\varphi(N)}{|\Delta|} \frac{|\Delta|}{\left|\pi_{d}(\Delta)\right|} \frac{1}{(N / d, d)} \varphi((d, N / d)) \\
& =\sum_{\substack{d \mid N \\
d>0}} \varphi(d) \varphi(N / d) /\left|\pi_{d}(\Delta)\right| .
\end{aligned}
$$


The last equality can be shown by using the fact that

$$
\varphi\left(n_{1}\right) \varphi\left(n_{2}\right)=\varphi\left(n_{1} n_{2}\right) \frac{\varphi\left(\left(n_{1}, n_{2}\right)\right)}{\left(n_{1}, n_{2}\right)}
$$

2. Hyperelliptic modular curves. If a curve $X$ is 2 -gonal, we call it sub-hyperelliptic. Also if $X$ is sub-hyperelliptic of genus $g \geq 2$, then it is called hyperelliptic.

Proposition 2.1 ([Ne, N-S]). Let $X_{1}$ and $X_{2}$ be smooth projective curves over an algebraically closed field $k$, and assume that there is a finite morphism $X_{1} \rightarrow X_{2}$ over $k$. If $X_{1}$ is d-gonal, so is $X_{2}$.

The best general lower bound for the gonality of a modular curve seems to be the one that is obtained in the following way.

Let $\lambda_{1}$ be the smallest positive eigenvalue of the Laplacian operator on the Hilbert space $L^{2}\left(X_{\Gamma}\right)$ where $X_{\Gamma}$ is the modular curve corresponding to a congruence subgroup $\Gamma$ of $\Gamma(1)$, and let $D_{\Gamma}$ be the index of $\bar{\Gamma}$ in $\bar{\Gamma}(1)$. Abramovich $[\mathrm{A}]$ shows the following inequality:

$$
\lambda_{1} D_{\Gamma} \leq 24 \operatorname{Gon}\left(X_{\Gamma}\right) .
$$

Using the best known lower bound for $\lambda_{1}$, due to Henry Kim and Peter Sarnak, as reported on page 18 of [B-G-G-P], i.e., $\lambda_{1}>0.238$, we get the following result.

THEOREM 2.2. Let $X_{\Gamma}$ be the modular curve corresponding to a congruence subgroup $\Gamma$ of index $D_{\Gamma}:=[\bar{\Gamma}(1): \bar{\Gamma}]$. Then

$$
D_{\Gamma}<\frac{12000}{119} \operatorname{Gon}\left(X_{\Gamma}\right) .
$$

In the following, we call the inequality in Theorem 2.2 Abramovich's bound.

Ishii and Momose [I-M] asserted that there existed no hyperelliptic modular curves $X_{\Delta}(N)$ with $\{ \pm 1\} \varsubsetneqq \Delta \varsubsetneqq(\mathbb{Z} / N \mathbb{Z})^{*}$. But we get the following result.

THEOREM 2.3. There exists a unique hyperelliptic modular curve of the form $X_{\Delta}(N)$ with $\{ \pm 1\} \varsubsetneqq \Delta \varsubsetneqq(\mathbb{Z} / N \mathbb{Z})^{*}$, namely $X_{\Delta_{1}}(21)$ where $\Delta_{1}$ is in Table 1.

REMARK 2.4. In [I-M] the mistake concerned Atkin-Lehner involutions on $X_{\Delta}(N)$. The Atkin-Lehner involutions define a unique involution on $X_{0}(N)$ but this does not hold for $X_{\Delta}(N)$.

To prove Theorem 2.3, we need some preparations.

Let $X$ be a smooth projective curve of genus $g \geq 2$ and $\Omega^{1}(X)$ the space of holomorphic differential forms on $X$. Then $\Omega^{1}(X)$ gives rise to a 
line bundle, called the canonical bundle, which in certain situations gives an embedding into projective space.

Let $\omega_{1}, \ldots, \omega_{g}$ be a basis for $\Omega^{1}(X)$. Viewing $X$ as a Riemann surface, we may choose a finite covering of open sets, with local parameters $z$ on each set, such that we can locally write $\omega_{i}=f_{i}(z) d z$. Then we get the well-defined map

$$
\phi: X \rightarrow \mathbb{P}^{g-1}, \quad P \mapsto\left(\omega_{1}(P): \cdots: \omega_{g}(P)\right) .
$$

Note that $\left(\omega_{1}(P): \cdots: \omega_{g}(P)\right)=\left(f_{1}(P): \cdots: f_{g}(P)\right)$. The above map is called the canonical map. Let $\bar{X}$ denote the image of $X$ under the canonical map. It is well-known that if $X$ is not hyperelliptic then the canonical map is injective.

If $X$ is a hyperelliptic curve of genus $g \geq 3$, then the image $\bar{X}$ under the canonical map is a smooth curve which is isomorphic to $\mathbb{P}^{1}$ and which is described by $(g-1)(g-2) / 2$ quadratic equations (see $\S 2$ of [Ga]).

Therefore it is possible to distinguish between hyperelliptic and nonhyperelliptic curves by examining their images under the canonical map.

Now we consider the modular curves $X_{\Delta}(N)$ of genus $g=g_{\Delta}(N) \geq 3$. Let $S_{\Delta}^{2}(N)$ denote the space of cusp forms of weight 2 . Suppose $\left\{f_{1}, \ldots, f_{g}\right\}$ is a basis of $S_{\Delta}^{2}(N)$. Then the canonical map may be written as

$$
X_{\Delta}(N) \ni P \mapsto\left(f_{1}(P): \cdots: f_{g}(P)\right) \in \mathbb{P}^{g-1} .
$$

One can get such a basis and their Fourier coefficients from [St]. Then to obtain a system of quadratic generators of $I\left(\overline{X_{\Delta}(N)}\right)$, we only have to compute the relations of the $f_{i} f_{j}(1 \leq i, j \leq g)$. If $X_{\Delta}(N)$ is not hyperelliptic, then there exist exactly $(g-2)(g-3) / 2$ linear relations among the $f_{i} f_{j}$ (see $\S 2$ of [H-S1]).

Now we are ready to prove Theorem 2.3. By Proposition 2.1 it suffices to consider $X_{\Delta}(N)$ when $X_{0}(N)$ is sub-hyperelliptic. If $g_{0}(N) \leq 2$, then one can find all $X_{\Delta}(N)$ for such $N$ in Table 1 . The other cases can be found in Table 2.

First applying Abramovich's bound we get the following result:

Lemma 2.5. The modular curves $X_{\Delta_{i}^{\dagger}}$ and $X_{\Delta_{i}^{\ddagger}}$ in Tables 1 and 2 are not hyperelliptic.

Remark 2.6. The notations $\Delta_{i}^{\dagger}$ and $\Delta_{i}^{\ddagger}$ in the tables mean that Abramovich's bound does not hold for $X_{\Delta_{i}^{\dagger}}(N)$ and $X_{\Delta_{i}^{\ddagger}}(N)$ when $\operatorname{Gon}\left(X_{\Gamma_{\Delta_{i}^{\dagger}}(N)}\right)$ $\leq 2$ and $\operatorname{Gon}\left(X_{\Gamma_{\Delta_{i}^{\ddagger}}(N)}\right) \leq 3$ respectively.

Now we prove that $X_{\Delta_{1}}(21)$ is a hyperelliptic curve in two different ways. 
Proof 1. The space $S_{\Delta_{1}}^{2}(21)$ is of dimension 3 and from [St] we can get a basis consisting of three newforms, as follows:

$$
\begin{aligned}
& f_{1}=q-q^{2}+q^{3}-q^{4}-2 q^{5}-q^{6}-q^{7}+3 q^{8}+q^{9}+2 q^{10}+\cdots \\
& f_{2}=q-q^{3}-2 q^{4}+2 q^{6}-2 q^{7}+4 q^{10}+2 q^{11}+q^{13}-2 q^{14}-\cdots \\
& f_{3}=2 q^{2}-q^{3}-2 q^{4}-2 q^{5}+q^{7}+q^{9}+4 q^{10}+2 q^{11}+q^{13}-\cdots
\end{aligned}
$$

By using the computer algebra system MAPLE we get a quadratic generator of the ideal $I\left(\overline{X_{\Delta_{1}}(21)}\right)$ :

$$
Q: x_{1}^{2}-x_{2}^{2}-x_{3}^{2}+x_{2} x_{3}
$$

where we obtain the relation $Q\left(f_{1}, f_{2}, f_{3}\right)=0$ by assigning $x_{i}$ to $f_{i}$. But this means that $X_{\Delta_{1}}(21)$ is hyperelliptic by the above criterion.

Proof 2. In [J-K1] it is proved that $X_{1}(21)$ is bielliptic, i.e., it admits a map of degree 2 to an elliptic curve, and all the bielliptic involutions on $X_{1}(21)$ are $W_{3}=\left(\begin{array}{cc}9 & -4 \\ 21 & -9\end{array}\right)$ and $[8] W_{3}$ where $[a]$ denotes the automorphism of $X_{1}(N)$ represented by $\gamma \in \Gamma_{0}(N)$ such that $\gamma \equiv\left(\begin{array}{l}a * \\ 0\end{array}\right) \bmod N$. Note that for bielliptic curves of genus 5 all bielliptic involutions commute with each other [Sch, Lemma 4.4]. Let $G$ be the group generated by the two bielliptic involutions of $X_{1}(21)$. Then we can determine the genus of the quotient $G \backslash X_{1}(21)$ by the four-group rule $[\mathrm{F}]$ as follows:

$$
\begin{aligned}
g\left(X_{1}(21)\right)= & g\left(W_{3} \backslash X_{1}(21)\right)+g\left([8] W_{3} \backslash X_{1}(21)\right) \\
& +g\left([8] \backslash X_{1}(21)\right)-2 g\left(G \backslash X_{1}(21)\right) .
\end{aligned}
$$

Thus $G \backslash X_{1}(21)$ is rational, and hence we get a Galois covering $X_{1}(21) \rightarrow \mathbb{P}^{1}$ with Galois group $G$. Since [8] \} X _ { 1 } ( 2 1 ) \text { is the same as } X _ { \Delta _ { 1 } } ( 2 1 ) \text { , we conclude } that $X_{\Delta_{1}}(21)$ is hyperelliptic.

To show that no other curve $X_{\Delta}(N)$ is hyperelliptic it suffices to consider $X_{\Delta}(N)$ for the maximal subgroups $\Delta$. For example, the modular curve $X_{\Delta_{1}}(30)$ is of genus 5 and has a basis of $S_{\Delta_{1}}^{2}(30)$ which consists of two old forms and three new forms:

$$
\begin{aligned}
& f_{1}=q-q^{2}-q^{3}-q^{4}+q^{5}+q^{6}+3 q^{8}+q^{9}-q^{10}-\cdots \\
& f_{2}=q^{2}-q^{4}-q^{6}-q^{8}+q^{10}+q^{12}+3 q^{16}+q^{18}-q^{20}-\cdots \\
& f_{3}=q-q^{2}+q^{3}+q^{4}-q^{5}-q^{6}-4 q^{7}-q^{8}+q^{9}+q^{10}+\cdots \\
& f_{4}=q-q^{4}-2 q^{5}+q^{6}-q^{9}-q^{10}+2 q^{11}+2 q^{14}+q^{15}+\cdots \\
& f_{5}=q^{2}-q^{3}+q^{5}-2 q^{7}-q^{8}-2 q^{10}+q^{12}+6 q^{13}+2 q^{15}-\cdots
\end{aligned}
$$


By using MAPLE we get three quadratic generators of $I\left(\overline{X_{\Delta_{1}}(30)}\right)$ :

$$
\left\{\begin{array}{l}
x_{4}^{2}-x_{5}^{2}-x_{1} x_{3}+2 x_{2} x_{3}-4 x_{4} x_{5} \\
x_{3}^{2}-2 x_{5}^{2}+2 x_{1} x_{2}-x_{1} x_{3}+2 x_{2} x_{3}-4 x_{4} x_{5} \\
x_{1}^{2}+4 x_{2}^{2}-2 x_{5}^{2}+2 x_{1} x_{2}-x_{1} x_{3}+2 x_{2} x_{3}-4 x_{4} x_{5} .
\end{array}\right.
$$

This means that $X_{\Delta_{1}}(30)$ is not hyperelliptic. A case by case calculation of the quadratic generators of $I\left(\overline{X_{\Delta}(N)}\right)$ for maximal subgroups $\Delta$ in Tables 1 and 2 finishes the proof of Theorem 2.3.

3. Trigonal modular curves. In this section we determine all trigonal modular curves $X_{\Delta}(N)$. Combining Theorem 2.3 with Proposition 2.1 it suffices to consider the modular curves $X_{\Delta}(N)$ with $g_{\Delta}(N) \geq 5$ in Tables 1 and 3 which contain all the intermediate modular curves between $X_{1}(N)$ and $X_{0}(N)$ such that $X_{0}(N)$ is trigonal.

Applying Abramovich's bound we get the following result.

Lemma 3.1. None of the modular curves $X_{\Delta_{i}^{\ddagger}}(N)$ in Tables 1 and 3 is trigonal.

We make use of the method due to Hasegawa and Shimura [H-S1].

TheOREm 3.2 (Petri's theorem). Let $X$ be a canonical curve of genus $g \geq 4$ defined over an algebraically closed field. Then the ideal $I(X)$ of $X$ is generated by some quadratic polynomials, unless $X$ is trigonal or isomorphic to a smooth plane quintic curve, in which cases it is generated by some quadratic and (at least one) cubic polynomials.

Let $X_{\Delta}(N)$ be of genus $g_{\Delta}(N) \geq 5$ and $\left\{f_{1}, \ldots, f_{g}\right\}$ a basis of $S_{\Delta}^{2}(N)$. Then to obtain a minimal generating system of the ideal $I\left(\overline{X_{\Delta}(N)}\right)$, we only have to compute the relations of the $f_{i} f_{j}$ and the $f_{i} f_{j} f_{k}(1 \leq i, j, k \leq g)$, and to eliminate those cubic relations arising from quadratic relations. By Petri's theorem, $X_{\Delta}(N)$ is trigonal if and only if it is not isomorphic to a smooth plane quintic curve, and a minimal generating system of $I\left(\overline{X_{\Delta}(N)}\right)$ contains a cubic polynomial. Let $Q_{1}, \ldots, Q_{(g-2)(g-3) / 2}$ be a system of quadratic generators of $I\left(\overline{X_{\Delta}(N)}\right)$. Since there are $(g-3)\left(g^{2}+6 g-10\right) / 6$ linear relations among the $f_{i} f_{j} f_{k}$, the number of cubic generators among the minimal generating system is

$$
\frac{(g-3)\left(g^{2}+6 g-10\right)}{6}-\operatorname{dim} L^{\prime}
$$

where $L^{\prime}$ is generated by $x_{i} Q_{j}(1 \leq i \leq g ; 1 \leq j \leq(g-2)(g-3) / 2)$. Thus $X_{\Delta}(N)$ is trigonal only if the above difference is non-zero.

ExAMPLE 3.3. The curve $X_{\Delta_{1}}(32)$ is of genus 5 and not hyperelliptic. By the exact same method as in the computation of $X_{\Delta_{1}}(30)$ ( see $\S 2$ ) we 
get three quadratic generators of $I\left(\overline{X_{\Delta_{1}}(32)}\right)$ :

$$
\left\{\begin{array}{l}
x_{1}^{2}+x_{2}^{2}+x_{3}^{2}+8 x_{5}^{2}+2 x_{2} x_{3}+4 x_{2} x_{4}-4 x_{2} x_{5}-8 x_{4} x_{5}, \\
-x_{2} x_{3}-x_{2} x_{4}-x_{2} x_{5}-x_{3} x_{4}+x_{3} x_{5} \\
x_{4}^{2}-x_{5}^{2}+x_{2} x_{5}+x_{3} x_{4}+2 x_{4} x_{5}
\end{array}\right.
$$

By a simple calculation we find that the dimension of $L^{\prime}$ is exactly 15 ; it follows that there are no essential cubic generators. Therefore $X_{\Delta_{1}}(30)$ is not trigonal.

Following the same method as in the above example we calculate the remaining cases to get the following result.

THEOREM 3.4. The modular curve $X_{\Delta}(N)$ is trigonal if and only if it is of genus $g_{\Delta}(N) \leq 2$ or not hyperelliptic with $g_{\Delta}(N)=3,4$. This happens exactly for all the curves $X_{\Delta}(N)$ of genus $g_{\Delta}(N) \leq 4$ in Table 1 except $X_{\Delta_{1}}(21)$.

Acknowledgments. We thank Andreas Schweizer for suggesting the second proof of Theorem 2.3. We also thank Enrique González-Jiménez for the comment on the lost hyperelliptic curve $X_{\Delta_{1}}(21)$.

\section{Appendix}

Table 1. List of $X_{\Delta}(N)$ and their genera $g_{\Delta}(N)$ when $X_{0}(N)$ are of genus $g_{0}(N) \leq 2$

\begin{tabular}{clc}
\hline$N$ & $\{ \pm 1\} \subsetneq \Delta \subsetneq(\mathbb{Z} / N \mathbb{Z})^{*}$ & $g_{\Delta}(N)$ \\
\hline $1 \leq N \leq 12$ & - & - \\
13 & $\Delta_{1}=\{ \pm 1, \pm 5\}$ & 0 \\
13 & $\Delta_{2}=\{ \pm 1, \pm 3, \pm 4\}$ & 0 \\
14 & - & - \\
15 & $\Delta_{1}=\{ \pm 1, \pm 4\}$ & 1 \\
16 & $\Delta_{1}=\{ \pm 1, \pm 7\}$ & 0 \\
17 & $\Delta_{1}=\{ \pm 1, \pm 4\}$ & 1 \\
17 & $\Delta_{2}=\{ \pm 1, \pm 2, \pm 4, \pm 8\}$ & 1 \\
18 & - & - \\
19 & $\Delta_{1}=\{ \pm 1, \pm 7, \pm 8\}$ & 1 \\
20 & $\Delta_{1}=\{ \pm 1, \pm 9\}$ & 1 \\
21 & $\Delta_{1}=\{ \pm 1, \pm 8\}$ & 3 \\
21 & $\Delta_{2}=\{ \pm 1, \pm 4, \pm 5\}$ & 1 \\
22 & - & - \\
23 & - & - \\
24 & $\Delta_{1}=\{ \pm 1, \pm 5\}$ & 3 \\
24 & $\Delta_{2}=\{ \pm 1, \pm 7\}$ & 3 \\
\hline
\end{tabular}


Table 1 (cont.)

\begin{tabular}{|c|c|c|}
\hline$N$ & $\{ \pm 1\} \subsetneq \Delta \subsetneq(\mathbb{Z} / N \mathbb{Z})^{*}$ & $g_{\Delta}(N)$ \\
\hline 24 & $\Delta_{3}=\{ \pm 1, \pm 11\}$ & 1 \\
\hline 25 & $\Delta_{1}=\{ \pm 1, \pm 7\}$ & 4 \\
\hline 25 & $\Delta_{2}=\{ \pm 1, \pm 4, \pm 6, \pm 9, \pm 11\}$ & 0 \\
\hline 26 & $\Delta_{1}=\{ \pm 1, \pm 5\}$ & 4 \\
\hline 26 & $\Delta_{2}=\{ \pm 1, \pm 3, \pm 9\}$ & 4 \\
\hline 27 & $\Delta_{1}=\{ \pm 1, \pm 8, \pm 10\}$ & 1 \\
\hline 28 & $\Delta_{1}=\{ \pm 1, \pm 13\}$ & 4 \\
\hline 28 & $\Delta_{2}=\{ \pm 1, \pm 3, \pm 9\}$ & 4 \\
\hline 29 & $\Delta_{1}^{\dagger}=\{ \pm 1, \pm 12\}$ & 8 \\
\hline 29 & $\Delta_{2}=\{ \pm 1, \pm 4, \pm 5, \pm 6, \pm 7, \pm 9, \pm 13\}$ & 4 \\
\hline 31 & $\Delta_{1}=\{ \pm 1, \pm 5, \pm 6\}$ & 6 \\
\hline 31 & $\Delta_{2}=\{ \pm 1, \pm 2, \pm 4, \pm 8, \pm 15\}$ & 6 \\
\hline 32 & $\Delta_{1}=\{ \pm 1, \pm 15\}$ & 5 \\
\hline 32 & $\Delta_{2}=\{ \pm 1, \pm 7, \pm 9, \pm 15\}$ & 1 \\
\hline 36 & $\Delta_{1}^{\dagger}=\{ \pm 1, \pm 17\}$ & 7 \\
\hline 36 & $\Delta_{2}=\{ \pm 1, \pm 11, \pm 13\}$ & 3 \\
\hline 37 & $\Delta_{1}^{\ddagger}=\{ \pm 1, \pm 6\}$ & 16 \\
\hline 37 & $\Delta_{2}^{\dagger}=\{ \pm 1, \pm 10, \pm 11\}$ & 10 \\
\hline 37 & $\Delta_{3}=\{ \pm 1, \pm 6, \pm 8, \pm 10, \pm 11, \pm 14\}$ & 4 \\
\hline 37 & $\Delta_{4}=\{ \pm 1, \pm 3, \pm 4, \pm 7, \pm 9, \pm 10, \pm 11, \pm 12, \pm 16\}$ & 4 \\
\hline 49 & $\Delta_{1}^{\ddagger}=\{ \pm 1, \pm 18, \pm 19\}$ & 19 \\
\hline 49 & $\Delta_{2}=\{ \pm 1, \pm 6, \pm 8, \pm 13, \pm 15, \pm 20, \pm 22\}$ & 3 \\
\hline 50 & $\Delta_{1}^{\ddagger}=\{ \pm 1, \pm 7\}$ & 22 \\
\hline 50 & $\Delta_{2}=\{ \pm 1, \pm 9, \pm 11, \pm 19, \pm 21\}$ & 4 \\
\hline
\end{tabular}

Table 2. List of $X_{\Delta}(N)$ and their genera $g_{\Delta}(N)$ when $X_{0}(N)$ are hyperelliptic and $g_{0}(N)>2$

\begin{tabular}{llc}
\hline$N$ & $\{ \pm 1\} \subsetneq \Delta \subsetneq(\mathbb{Z} / N \mathbb{Z})^{*}$ & $g_{\Delta}(N)$ \\
\hline 30 & $\Delta_{1}=\{ \pm 1, \pm 11\}$ & 5 \\
33 & $\Delta_{1}^{\dagger}=\{ \pm 1, \pm 10\}$ & 11 \\
33 & $\Delta_{2}=\{ \pm 1, \pm 2, \pm 4, \pm 8, \pm 16\}$ & 5 \\
35 & $\Delta_{1}^{\dagger}=\{ \pm 1, \pm 6\}$ & 13 \\
35 & $\Delta_{2}=\{ \pm 1, \pm 11, \pm 16\}$ & 9 \\
35 & $\Delta_{3}=\{ \pm 1, \pm 6, \pm 8, \pm 13\}$ & 7 \\
35 & $\Delta_{4}=\{ \pm 1, \pm 4, \pm 6, \pm 9, \pm 11, \pm 16\}$ & 5 \\
39 & $\Delta_{1}^{\dagger}=\{ \pm 1, \pm 14\}$ & 17 \\
39 & $\Delta_{2}^{\dagger}=\{ \pm 1, \pm 16, \pm 17\}$ & 9 \\
39 & $\Delta_{3}=\{ \pm 1, \pm 5, \pm 8, \pm 14\}$ & 9 \\
\hline
\end{tabular}


Table 2 (cont.)

\begin{tabular}{llc}
\hline$N$ & $\{ \pm 1\} \subsetneq \Delta \subsetneq(\mathbb{Z} / N \mathbb{Z})^{*}$ & $g_{\Delta}(N)$ \\
\hline 39 & $\Delta_{4}=\{ \pm 1, \pm 4, \pm 10, \pm 14, \pm 16, \pm 17\}$ & 5 \\
40 & $\Delta_{1}^{\dagger}=\{ \pm 1, \pm 31\}$ & 9 \\
40 & $\Delta_{2}^{\dagger}=\{ \pm 1, \pm 9\}$ & 13 \\
40 & $\Delta_{3}^{\dagger}=\{ \pm 1, \pm 11\}$ & 13 \\
40 & $\Delta_{4}=\{ \pm 1, \pm 9, \pm 11, \pm 19\}$ & 5 \\
40 & $\Delta_{5}=\{ \pm 1, \pm 3, \pm 9, \pm 13\}$ & 7 \\
40 & $\Delta_{6}=\{ \pm 1, \pm 7, \pm 9, \pm 17\}$ & 71 \\
41 & $\Delta_{1}^{\dagger}=\{ \pm 1, \pm 9\}$ & 11 \\
41 & $\Delta_{2}^{\dagger}=\{ \pm 1, \pm 3, \pm 9, \pm 14\}$ & 11 \\
41 & $\Delta_{3}=\{ \pm 1, \pm 4, \pm 10, \pm 16, \pm 18\}$ & 5 \\
41 & $\Delta_{4}=\{ \pm 1, \pm 2, \pm 4, \pm 5, \pm 8, \pm 9, \pm 10, \pm 16, \pm 18, \pm 20\}$ & - \\
46 & - & - \\
47 & - & 19 \\
48 & $\Delta_{1}^{\dagger}=\{ \pm 1, \pm 7\}$ & 19 \\
48 & $\Delta_{2}^{\dagger}=\{ \pm 1, \pm 17\}$ & 19 \\
48 & $\Delta_{3}^{\dagger}=\{ \pm 1, \pm 23\}$ & 5 \\
48 & $\Delta_{4}=\{ \pm 1, \pm 11, \pm 13, \pm 23\}$ & 7 \\
48 & $\Delta_{5}=\{ \pm 1, \pm 7, \pm 17, \pm 23\}$ & 7 \\
48 & $\Delta_{6}=\{ \pm 1, \pm 5, \pm 19, \pm 23\}$ & - \\
59 & - & 36 \\
71 & $\Delta_{1}^{\dagger}=\{ \pm 1, \pm 5, \pm 14, \pm 17, \pm 25\}$ & 26 \\
71 & $\Delta_{2}^{\dagger}=\{ \pm 1, \pm 20, \pm 23, \pm 26, \pm 30, \pm 32, \pm 34\}$ & \\
\hline & &
\end{tabular}

Table 3. List of $X_{\Delta}(N)$ and their genera $g_{\Delta}(N)$ when $X_{0}(N)$ are trigonal but not sub-hyperelliptic

\begin{tabular}{llc}
\hline$N$ & $\{ \pm 1\} \subsetneq \Delta \subsetneq(\mathbb{Z} / N \mathbb{Z})^{*}$ & $g_{\Delta}(N)$ \\
\hline 34 & $\Delta_{1}=\{ \pm 1, \pm 13\}$ & 9 \\
34 & $\Delta_{2}=\{ \pm 1, \pm 9, \pm 13, \pm 15\}$ & 5 \\
38 & $\Delta_{1}=\{ \pm 1, \pm 7, \pm 11\}$ & 10 \\
43 & $\Delta_{1}^{\ddagger}=\{ \pm 1, \pm 6, \pm 7\}$ & 15 \\
43 & $\Delta_{2}=\{ \pm 1, \pm 2, \pm 4, \pm 8, \pm 11, \pm 16, \pm 21, \pm 22\}$ & 9 \\
44 & $\Delta_{1}^{\ddagger}=\{ \pm 1, \pm 21\}$ & 16 \\
44 & $\Delta_{2}=\{ \pm 1, \pm 5, \pm 7, \pm 9, \pm 19\}$ & 8 \\
45 & $\Delta_{1}^{\ddagger}=\{ \pm 1, \pm 19\}$ & 21 \\
45 & $\Delta_{2}=\{ \pm 1, \pm 14, \pm 16\}$ & 9 \\
45 & $\Delta_{3}=\{ \pm 1, \pm 8, \pm 17, \pm 19\}$ & 11 \\
45 & $\Delta_{4}=\{ \pm 1, \pm 4, \pm 11, \pm 14, \pm 16, \pm 19\}$ & 5 \\
53 & $\Delta_{1}^{\ddagger}=\{ \pm 1, \pm 23\}$ & 40 \\
\hline
\end{tabular}


Table 3 (cont.)

\begin{tabular}{llc}
\hline$N$ & $\{ \pm 1\} \subsetneq \Delta \subsetneq(\mathbb{Z} / N \mathbb{Z})^{*}$ & $g_{\Delta}(N)$ \\
\hline 53 & $\Delta_{2}=\{ \pm 1, \pm 4, \pm 6, \pm 7, \pm 9, \pm 10, \pm 11, \pm 13, \pm 15, \pm 16, \pm 17, \pm 24, \pm 25\}$ & 8 \\
54 & $\Delta_{1}^{\ddagger}=\{ \pm 1, \pm 17, \pm 19\}$ & 10 \\
61 & $\Delta_{1}^{\ddagger}=\{ \pm 1, \pm 11\}$ & 56 \\
61 & $\Delta_{2}^{\ddagger}=\{ \pm 1, \pm 13, \pm 14\}$ & 36 \\
61 & $\Delta_{3}^{\ddagger}=\{ \pm 1, \pm 3, \pm 9, \pm 20, \pm 27\}$ & 26 \\
61 & $\Delta_{4}^{\ddagger}=\{ \pm 1, \pm 11, \pm 13, \pm 14, \pm 21, \pm 29\}$ & 16 \\
61 & $\Delta_{5}=\{ \pm 1, \pm 3, \pm 8, \pm 9, \pm 11, \pm 20, \pm 23, \pm 24, \pm 27, \pm 28\}$ & 12 \\
64 & $\Delta_{1}^{\ddagger}=\{ \pm 1, \pm 31\}$ & 37 \\
64 & $\Delta_{2}^{\ddagger}=\{ \pm 1, \pm 15, \pm 17, \pm 31\}$ & 13 \\
64 & $\Delta_{3}=\{ \pm 1, \pm 7, \pm 9, \pm 15, \pm 17, \pm 23, \pm 25, \pm 31\}$ & 5 \\
81 & $\Delta_{1}^{\ddagger}=\{ \pm 1, \pm 26, \pm 28\}$ & 46 \\
81 & $\Delta_{2}^{\ddagger}=\{ \pm 1, \pm 8, \pm 10, \pm 17, \pm 19, \pm 26, \pm 28, \pm 35, \pm 37\}$ & 10 \\
\hline
\end{tabular}

\section{References}

[A] D. Abramovich, A linear lower bound on the gonality of modular curves, Int. Math. Res. Not. 1996, no. 20, 1005-1011.

[B-G-G-P] M. H. Baker, E. González-Jiménez, J. González and B. Poonen, Finiteness results for modular curves of genus at least 2, Amer. J. Math. 127 (2005), 1325-1387.

[F] C. R. Ferenbaugh, The genus-zero problem for $n \mid h$-type groups, Duke Math. J. 72 (1993), 31-63.

[Ga] S. D. Galbraith, Equations for modular curves, D. Phil. Thesis, Oxford, 1996.

[H-S1] Y. Hasegawa and M. Shimura, Trigonal modular curves, Acta Arith. 88 (1999), 129-140.

[H-S2] - - - Trigonal modular curves $X_{0}^{+d}(N)$, Proc. Japan Acad. Ser. A Math. Sci. 75 (1999), no. 9, 172-175.

[H-S3] -, 一, Trigonal modular curves $X_{0}^{*}(N)$, ibid. 76 (2000), no. 6, 83-86.

[I-M] N. Ishii and F. Momose, Hyperelliptic modular curves, Tsukuba J. Math. 15 (1991), 413-423.

[J-K1] D. Jeon and C. H. Kim, Bielliptic modular curves $X_{1}(N)$, Acta Arith. 112 (2004), 75-86.

[J-K2] - - - Bielliptic modular curves $X_{1}(M, N)$, Manuscripta Math. 118 (2005), $455-466$.

[J-K-S] D. Jeon, C. H. Kim and A. Schweizer, On the torsion of elliptic curves over cubic number fields, Acta Arith. 113 (2004), 291-301.

[Ne] M. Newman, Conjugacy, genus and class number, Math. Ann. 196 (1972), 198-217.

[N-S] K. V. Nguyen and M.-H. Saito, d-gonality of modular curves and bounding torsions, math.AG/9603024.

[O1] A. Ogg, Modular Forms and Dirichlet Series, Benjamin, 1969.

[O2] - Rational points on certain elliptic modular curves, in: Proc. Sympos. Pure Math. 24, Amer. Math. Soc., 1973, 221-231. 
[Sch] A. Schweizer, Bielliptic Drinfeld modular curves, Asian J. Math. 5 (2001), 705-720.

[St] W. A. Stein, http://modular.fas.harvard.edu.

Department of Mathematics Education

Kongju National University

182 Shinkwan-dong

Kongju, Chungnam, 314-701 Korea

E-mail: dyjeon@kongju.ac.kr
Department of Mathematics Seoul Women's University 126 Kongnung 2-dong, Nowon-gu Seoul, 139-774 Korea E-mail: chkim@swu.ac.kr 